\title{
Case Report of Combined Twin Tubal and Intrauterine Pregnancies
}

Ospan A Mynbaev ${ }^{1,2 *}$, Artur K Khachatryan ${ }^{2,3}$, Ioannis P Kosmas ${ }^{4}$, Marina Yu Eliseeva ${ }^{2,5}$, A Khachatryan ${ }^{2}$, Antonio Malvasi ${ }^{6}$ and Andrea Tinelli $^{7}$

${ }^{1}$ The Experimental Research and Modelling Division, and the Department of Obstetrics and Gynecology, Moscow State University of Medicine and Dentistry, Moscow, Russia

${ }^{2}$ The Department of Obstetrics and Gynecology and Reproductive Medicine, The Russian University of Peoples' Friendship, Moscow, Russia

${ }^{3}$ N79 City Clinical Hospital, Moscow, Russia

${ }^{4}$ Xatzikosta General Hospital, loannina, Greece

${ }^{5}$ The Institute of Reproductive Technologies, AltraVita, Moscow, Russia

${ }^{6}$ Department of Obstetrics and Gynecology, Division of Experimental Endoscopic Surgery, Imaging, Minimally Invasive Therapy and Technology, Italy

${ }^{7}$ Department of Obstetrics and Gynecology, Division of Experimental Endoscopic Surgery, Imaging, Minimally Invasive Therapy and Technology, Lecce, Italy

\section{Abstract}

Case of heterotopic quadruplet pregnancy with coexistence of tubal and intrauterine twins.

Objective (s): To present the case of heterotopic quadruplet pregnancy with coexistence of tubal and intrauterine twins and its successful surgical management with brief literature review.

Design: A case report supplemented with brief literature review and discussion.

Setting: City clinical university hospital.

Patient (s): Heterotopic quadruplet pregnancy with tubal and intrauterine twins in a 31-year-old woman after natural conception.

Intervention (s): Laparoscopy, adhesiolisys, aspiration of tubal embryos through tubotomy and cervical dilatation and vacuum aspiration of intrauterine embryos as well as vaginal and intraoperative laparoscopic ultrasound examination.

Main outcome measure (s): Physical examination, laparoscopy and postsurgical hCG serum assay, as well as preintra- and postoperative vaginal ultrasound and intraoperative laparoscopic sonography.

Result (s): Successful diagnosis and treatment of a case of heterotopic quadruplet pregnancy with coexistence of tubal and intrauterine twins in a 31-year-old woman with 6 artificial abortions, pelvic inflammatory diseases and periadnexal adhesions, after natural conception was described. An intraoperative laparoscopic sonography was applied to complete diagnosis of heterotopic pregnancy with coexistence of tubal and intrauterine twins after control of bleeding and stabilization of patient condition due to necessity to perform an emergency surgery. The case was supplemented with a brief review of literature and discussion.

Conclusion (s): A unique case of heterotopic quadruplet pregnancy with coexistence of tubal and intrauterine twins after natural conception was presented in the first time.

Keywords: Heterotopic quadruplet pregnancy; Intrauterine twins and tubal twins; Laparoscopic echography

\section{Introduction}

An increasing prevalence of Ectopic Pregnancies (EPs) is a rise concern, due to widespread application of the state-of-the art Assisted Reproductive Technologies (ARTs). There are also other risk factors of EP development, including sexually transmitted and pelvic inflammatory diseases (PIDs), specially adnexitis and salpingitis with formation of hydrosalpings, intraperitoneal and periadnexal adhesions and partial occlusion of Fallopian Tubes (FTs), as well as gynaecological surgical procedures, which may lead to tubal dysfunction and ectopic embryo implantation [1]. Moreover an increased prevalence of multiple pregnancies during conception after stimulated ovulation is multiplying risk of Heterotopic Pregnancy (HP), when ectopic and intrauterine pregnancies are simultaneously coexisted.

Several cases of HPs have reported even in patients without risk factors. A case of combined tubal and intrauterine pregnancies was reported by Varras et al. [2] in a 28-year-old woman after Natural Conception (NC), when an emergency Laparotomy (LT) with tubectomy was performed due to 1.5 liter haemoperitoneum. Bettocchi et al. [3] described a case report of simultaneous bilateral tubal pregnancies and intrauterine pregnancy with five embryos in 28-year-old women after stimulation of ovulation with gonadotropins and NC. HPs were removed by salpingotomy in the right FT and by salpingectomy in left FT, whereas multiple intrauterine pregnancies were terminated with two fetuses after selective embryo reduction [3]. A case of combined triplet HP with twin tubal and singlet intrauterine pregnancies after NC was reported by Simsek et al [4] in 37-year-old patient. LT and tubectomy was performed due to twin tubal pregnancy with a right ruptured FT and intrauterine pregnancy was terminated without complications. Jibodu and Darne [5] presented a case of combined tubal and intrauterine singleton pregnancies in a 29 -yearold woman after NC.

This report aims to show in the first time a unique case of heterotopic quadruplet pregnancy with coexistence of tubal and intrauterine twins in a 31-year-old woman after NC with brief review of literature. The intraoperative laparoscopic sonography was successfully applied to properly visualize both tubal and intrauterine twins as well as to monitor the effectiveness of surgical treatment.

\section{Materials and Methods}

A case presents a 31-year-old woman with heterotopic quadruplet pregnancy coexisting tubal and intrauterine twins after natural

*Corresponding author: Dr. OA Mynbaev, Professor, The Department of Obstetrics and Gynecology and Reproductive Medicine, Head, The Experimenta Researches and Modeling Division, Box. No. 19.15, Delegatskaya str, 20/1 Moscow, 127374, Russia, Tel: +79161271546; E-mail: ospanmynbaev@hotmail.com

Received December 10, 2012; Accepted January 15, 2013; Published January 17,2013

Citation: Mynbaev OA, Khachatryan AK, Kosmas IP, Eliseeva MY, Khachatryan TA, et al. (2013) Case Report of Combined Twin Tubal and Intrauterine Pregnancies. J Clin Case Rep 3: 242. doi:10.4172/2165-7920.1000242

Copyright: (c) 2013 Mynbaev OA, et al. This is an open-access article distributed under the terms of the Creative Commons Attribution License, which permits unrestricted use, distribution, and reproduction in any medium, provided the original author and source are credited. 
conception. The patient signed a written consent for emergency surgery with application of an intraoperative laparoscopic sonography as well as a request for the vacuum aspiration and removal of the intrauterine embryos.

37 original reports with 46 cases of coexistence of tubal and intrauterine pregnancies were available for review in MEDLINE database. Only reports concerning HPs coexisting tubal and intrauterine embryos were reviewed. HPs with interstitial and other locations of embryos were excluded. Way of conception, number and location of coexisting embryos, condition of uterine tubes and volume of hemoperitoneum, type of surgical procedures and outcome of intrauterine pregnancies were analyzed.

\section{Case Report}

A 31-year-old patient was admitted to the gynecological department as an emergency case with complains about pain in her lower right abdomen, breast tenderness, nausea, vomiting and delayed menstruation over a period of 56 days.

Analysis of anamnesis showed that the patient had had regular menstruations for 5-6 days every 25-26 days starting from 13 years of age. Patient had had 7 pregnancies with only one term delivery and 6 artificial abortions. Patient had also experienced PIDs because of complications of artificial abortions. There were not cases of multiple pregnancies in both families of our patient and her husband.

A month before, a scanty dark brown discharge was displayed during 5 days at the time of expected menstruations. The patient first noticed a pain in her lower right abdomen 7 days before her admission and a 7-8 week pregnancy with miscarriage had been diagnosed during the first checkup at the out-patients' clinic by physical and vaginal examinations. Doctors had prescribed antispasmodic medications and rest. Since then, other symptoms of pregnancy had manifested and the pain had progressed in spite of treatment and rest.

The day of admission, the patient's condition had deteriorated and she had turned to the out-patients' clinic again, where the HP with single embryo in the right FT and another single intrauterine embryo was diagnosed. Vaginal examination caused sharp and severe pain and the patient was immediately hospitalized for emergency surgical treatment.

Gynecological examination at the moment of admission showed a presence of intrauterine pregnancy with a 8 week pregnancy size of the gravid uterus and a $4 \mathrm{~cm}$-diameter enlarged right FT, of tightly-elastic consistence and sharply painful during both palpation and ultrasound examination. Combined intrauterine and tubal embryos were found by transvaginal ultrasound examination: a $2.2 \mathrm{~cm}$ size intrauterine embryo and enlarged right FT in the middle part $(6.0 \times 3.2 \times 4.9 \mathrm{~cm})$ with a $2.1 \mathrm{~cm}$-size of an embryo (Figure 1). A hemoperitoneum with grumes were visualized in the abdominal and pelvic cavities.

The patient was immediately operated by means of the laparoscopic set of the company Karl Storz (Germany). Laparoscopic examination revealed about $350 \mathrm{~mL}$ of hemoperitoneum with clots and grumes in the abdominal and pelvic cavities with an enlarged gravid uterus, approximately of an 8-9 week pregnancy with a ball-like form. There was also enlarged unrupted right FT about 4-cm-diameter in diameter with a dark blue colour and its ampullar part was imbibed with leaking blood. The corpus luteus was situated in the left ovary and no changes were found in the right ovary and the left FT. There were also tuboperitoneal adhesions, involving the right ovary and FT.
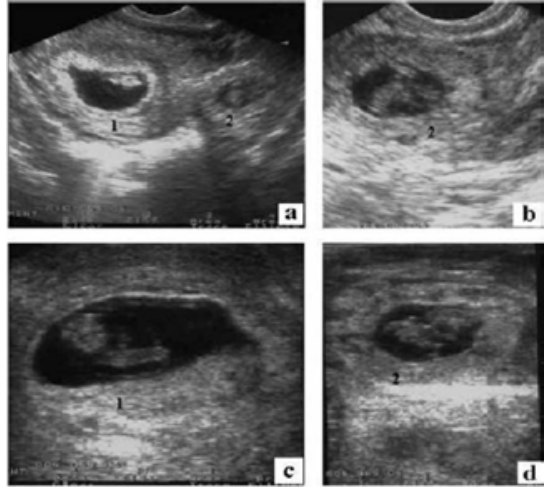

Figure 1: Ultrasound scan of heterotopic pregnancy by vaginal probe (a) intrauterine embryo, (b) tubal embryo and intraoperative laparoscopic probe (c) intrauterine twins and (d) tubal twins.

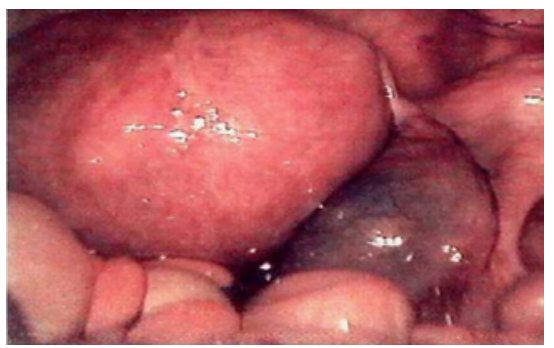

Figure 2: Laparoscopic picture of combined intrauterine and tubal pregnancies

Free blood in the abdominal and pelvic cavities was aspirated and blood clots and grumes were removed. After adhesiolysis corpus of uterus and both ovaries and FTs were visualized (Figure 2) and the situation was stabilized without life-threatening condition.

In our operation theatre a laparoscopic ultrasound probe had applied for all surgeries to investigate its diagnostic value and to control an efficiency of surgical procedures. Subsequently we performed laparoscopic sonography to distinguish location and number of heterotopic location of embryos by means of the ultrasound device "Aloka SSD-900" (Japan) and Siemens PRIMA, SIENA (Germany) with laparoscopic linear sensors with frequency $7.0 \mathrm{MHz}$ and irrigation of pelvic cavity with isotonic solution for ultrasound contrasting.

Laparoscopic sonography revealed heterotopic quadruplet pregnancy with coexistence of monochorionic and monoamniotic tubal and intrauterine twins (Figure 1). A development stage of the embryos was 7 weeks and 2 days. Double heart rates were registered in both tubal and intrauterine embryos about 152 and 146 strokes per min respectively. The chorionic thickness was $0.6-0.7 \mathrm{~cm}$ in both the uterine and tubal pregnancies. The size of intrauterine embryos was similar $(2.2 \mathrm{~cm})$, whereas tubal twins were different in their size $(2.1$ and $2.2 \mathrm{~cm}$ ).

All embryos were removed, the ectopic embryos by tubotomy and aspiration, whereas the intrauterine embryos through cervical dilatation till $\mathrm{N} 9.5$ by vacuum aspiration with cannula N 9. During surgery an ultrasound monitoring was done by laparoscopic probe with isotonic solution irrigation into pelvic cavity and FT, in order to make sure that all embryos and chorionic tissues had been completely eliminated.

We had not evaluated serum or urine hCG concentration before 
surgery because patient had been urgently admitted to hospital and emergency surgery had been performed. However, this assay was performed on the $5^{\text {th }}, 10^{\text {th }}$ and $15^{\text {th }}$ day after surgery showing a decrease in serum hCG concentrations resulting in 2904, 229 and $145 \mathrm{mIU} / \mathrm{ML}$ respectively due to performed conservative treatment. Immunoenzyme assay was done on the device "Cobas Core" of company Hoffman La Roche (Switzerland) with chemical reagents of company "DIA plus" (Russia). Diagnosis of coexistence of tubal and intrauterine twins was confirmed by histological examination.

Physical and ultrasound examination follow-up, one month after surgery, showed usual uterus and ovaries condition and size as well as an absence of pathological changes in the FTs.

\section{Discussion}

To our knowledge, this is the first report of a unique case of heterotopic quadruplet pregnancy with coexistence of tubal and intrauterine twins after NC. Although the prevalence of HPs with multiple embryos is rising due to widespread application of ARTs [1], spontaneously occurred HPs are remaining very rare. Therefore this unique case is represent the scientifically and practically significance to be analyzed in detail.

There were not cases of multiple pregnancies in both our patient's and her husband's families. In the past our patient had experienced 6 artificial abortions with subsequent manifestations of PIDs with formation of periadnexal adhesions which were eliminated during present surgery. Probably these factors were the main reasons of a spontaneously occurred HP. In this case the final diagnosis of HP with coexistence of tubal and intrauterine twins was successfully completed by means of laparoscopic US. We realize that a TV US is also an effective tool to detect these twin embryos in both intrauterine and tubal locations. Shortcomings of TV US in detection of these quadruplets in our case were related with sharp and severe pain during vaginal examination and possibility to increase bleeding and obvious indications to urgent surgery by clinical and TV US examinations. Since there was free hemoperitoneum, an evidence of both ectopic and intrauterine pregnancies the surgeon, who performed these US examinations, preferred, firstly to control of bleeding and stabilize the patient's condition and after that to repeat an US with laparoscopic probe to distinguish location and number of embryos, as well as to determine the size of damaged area of FT in order to perform conservative surgery. Laparoscopic US was also used to control an efficiency of surgical procedures, notably to visualize remaining parts of embryos and chorionic tissue in the fallopian tube and uterine cavity by liquid contrasting. Although abdominal and TV US was successfully applied in many cases of preoperative diagnosis of HPs [6,7], according to several reports abdominal and TV US scan failed to visualize tubal embryos in cases of HPs $[2,8,9]$ including partly its efficiency in reports with two cases $[10,11]$.

Among 37 reviewed reports in a seventuplet pregnancy, with bilateral tubal and five intrauterine embryos [3], in another sextuplet pregnancy with bilateral tubal and three intrauterine embryos [12], as well as a quadruplet pregnancy with single tubal and three intrauterine pregnancies [13] were coexisted. Intriguingly recurrent HPs after IVF attempts with involving both fallopian tubes by turns [11], when, during the first pregnancy a left tubectomy was performed with subsequent interruption of intrauterine pregnancy and during the second pregnancy a right a tubectomy was done in the sequel live birth twins. 12/37 reports presented triplets, where five intrauterine twins $[11,14-17]$ and three tubal twins $[4,7,18]$ were coexisted with vise- versa tubal or intrauterine single pregnancies. Interestingly there was coexistence of bilateral tubal and intrauterine single embryos in 4 cases among these triplets [19-22]. 25/37 reports described duplets, when 31 cases of HPs were presented with coexisting tubal and intrauterine single embryos [5,6,8,9,11,17,23-38]. 2/37 reports described two cases of HPs with both triplets and duplets [11,17], and 4/37 reports presented 2 cases with duplets $[10,24,26,29]$.

Thus we analyzed 46 cases of HP with different number of embryos and from this view, present report is introduced a unique case of coexistence of heterotopic quadruplet pregnancy with two twins and subsequently completed a mosaic appearance of possible combination of multiple tubal and intrauterine pregnancies. It is important to release all possible coexistence of implantation's locations due to an increased prevalence of ectopic and multiple pregnancies as well as HPs in a widespread ARTs' era.

Information was not available according to treatments before conception in 5/46 cases, and conception was followed after application of ARTs in 31/46 cases, including full cycle of IVF-ET protocols in 20 cases and a stimulation of ovulation with clomiphene citrate and other hormonal medications in 11 cases with a subsequent: NC in 6 cases; an intrauterine sperm injection in 5 cases. Subsequently there was NC in cycles without any treatment only in 10/46 cases, representing rare prevalence of HP after NC.

The condition of gravide FTs in 8/46 cases were not described. FTs were ruptured in 23/46 cases, they were distended and enlarged in 10/46 cases and leaking in 5/46 cases. There was hemiperitoneum in $28 / 46$ cases and amount of blood in the abdominal cavity was varied from 200 till $2300 \mathrm{~mL}$ with significantly reduced heamoglobin concentration and hematocrit rates in several cases, which requested hemotransfusion. These data recall us that a management of ectopic pregnancies, particulalrly HPs required efficiently make decision to perform emergency surgical preocedures to avoid lifetreatening condition.

Surgical procedures were done by LT for emergency reason in $26 / 46$ cases, by laparoscopy in $17 / 46$ cases and there was inversion from laparoscopy to LT in $2 / 46$ cases. There was not available information concerning type of surgery in $3 / 46$ cases. Radical surgery with salpingoectomy was performed in 36/46 cases, bilateral tubectomies in $2 / 46$ cases, and adnexectomy in $1 / 46$ case, whereas conservative surgery with tubotomy and aspiration of embryones and chorionic tissue was done in only $4 / 46$ cases. It shows that most cases surgeons used more traumatic for patients way of solution because of urgent character of situation, when patients were admired to the hospital or even when they were under observation well in advance of before surgery.

Outcomes of intrauterine pregnancies were not available in 10/46 reports. There were 27/46 live births with 9 twins and 18 single newborns. Intrauterine embryos were reduced in cases of multiple intraperitoneal pregnancies and remaining pregnancies were successfully terminated with live births $[3,12,13]$. Intrauterine pregnancies were interrupted by curettage or spontaneous abortion in $9 / 46$ cases $[11,19,21,22,26,29,30,37]$ and degeneration into vesicular mola was observed in $1 / 46$ case [31] .

In comparison with the data of literature the management of our patient with coexistence of intrauterine and tubal twins with leaking in extended right FT was successful with using only conservative surgical procedures, i.e, tubotomy and aspiration of embryos by laparoscopic approach and removal of intrauterine embryos since our patient did 
not want to preserve pregnancy. This method allowed us to control outcome of surgery and reduce time of surgery.

Thus, according to analysis of the rate of ruptured FTs followed with severe hemoperitoneum and the high frequency of urgently performed radical surgical procedures with hemotransfusions in this brief review a heterotopic tubal pregnancy is remaining as a risk factor for maternal morbidity and mortality. Therefore, in spite of improved management of ectopic pregnancies with highly effective laboratory and ultrasound diagnosis as well as laparoscopic treatment, an increasing prevalence of multiple and ectopic pregnancies is a rise concern of healthcare system, due to widespread application of the state-of-the art of ARTs with increased risk of heterotopic pregnancies.

\section{Conclusion}

A unique case of heterotopic quadruplet pregnancy with coexistence of tubal and intrauterine twins after natural conception in a 31-year-old woman with 6 artificial abortions, pelvic inflammatory diseases and periadnexal adhesions was reported. An intraoperative laparoscopic echography was applied to complete diagnosis of heterotopic pregnancy with coexistence of tubal and intrauterine twins after control of bleeding and stabilization of patient condition.

\section{References}

1. Velalopoulou A, Peschos D, Mynbaev O, Eliseeva M, Verginadis I et al. (2011) Ectopic pregnancy and assisted reproductive technologies: a systematic review. In bookR: Ectopic Pregnancy - Modern Diagnosis and Management.

2. Varras M, Akrivis C, Hadjopoulos G, Antoniou N (2003) Heterotopic pregnancy in a natural conception cycle presenting with tubal rupture: a case report and review of the literature. Eur J Obstet Gynecol Reprod Biol 106: 79-82.

3. Bettocchi S, Nappi L, Ceci O, Vimercati A, Selvaggi L, et al. (2004) Simultaneous bilateral tubal pregnancies and intrauterine pregnancy with five fetuses. J Am Assoc Gynecol Laparosc 11: 195-196.

4. Simsek T, Dogan A, Simsek M, Pestereli E (2008) Heterotopic triplet pregnancy (twin tubal) in a natural cycle with tubal rupture: case report and review of the literature. J Obstet Gynaecol Res 34: 759-762.

5. Jibodu OA, Darne FJ (1997) Spontaneous heterotopic pregnancy presenting with tubal rupture. Hum Reprod 12: 1098-1099.

6. Singhal M, Ahuja CK, Saxena AK, Dhaliwal L, Khandelwal N (2010) Sonographic appearance of heterotopic pregnancy with ruptured ectopic tubal pregnancy. $\mathrm{J}$ Clin Ultrasound 38: 509-511.

7. Goldman JA, Dicker D, Dekel A, Feldberg D, Ashkenazi J (1991) Successful management and outcome of heterotopic triplet in vitro fertilization (IVF) gestation: twin tubal and surviving intrauterine pregnancy. $J$ In Vitro Fert Embryo Transf 8: 300-302.

8. Anastasakis E, Jetti A, Macara L, Daskalakis G (2007) A case of heterotopic pregnancy in the absence of risk factors. A brief literature review. Fetal Diagn Ther 22: 285-288.

9. Noor N, Bano I, Parveen S (2012) Heterotopic pregnancy with successful pregnancy outcome. J Hum Reprod Sci 5: 213-214.

10. Barrenetxea G, Barinaga-Rementeria L, Lopez de LA, Agirregoikoa JA, Mandiola M, et al. (2007) Heterotopic pregnancy: two cases and a comparative review. Fertil Steril 87: 417-415.

11. Raziel A, Friedler S, Herman A, Strassburger D, Maymon R, et al. (1997) Recurrent heterotopic pregnancy after repeated in-vitro fertilization treatment. Hum Reprod 12: 1810-1812.

12. Fisher SL, Massie JA, Blumenfeld YJ, Lathi RB (2011) Sextuplet heterotopic pregnancy presenting as ovarian hyperstimulation syndrome and hemoperitoneum. Fertil Steril 95: 2431-2433.

13. Phipps WR, Evans MI (1991) Combined intrafallopian/intrauterine reduction of a quintuplet gestation. Fertil Steril 55: 1189-1191.
14. Cholkeri-Singh A, LaBarge A (2007) Spontaneous heterotopic triplets: a case report. Fertil Steril 88: 968-967.

15. Gözüküçük M, Çetinkaya SE, Aydınuraz B, Kahraman K, Şatıroğlu MH (2011) Live twin birth after successful treatment of a ruptured heterotopic pregnancy by laparoscopy. Gynecol Surg 8: 239-241.

16. Oliveira FG, Abdelmassih V, Abdelmassih OS, Abdelmassih R, Nagy ZP (2002) Heterotopic triplet pregnancy: report and video of a case of a ruptured tubal implantation with living embryo concurrent with an intrauterine twin gestation. Reprod Biomed Online 5: 313-316.

17. Gruber I, Lahodny J, Illmensee K, Losch A (2002) Heterotopic pregnancy: report of three cases. Wien Klin Wochenschr 114: 229-232.

18. Kasum M (2009) Unilateral twin tubal pregnancy and subseqyent heterotopic pregnancy in a patient following in vitro fertilization. Acta Clin Croat 48: 451454

19. Jeong HC, Park IH, Yoon YS, Lee NW, Kim HJ, et al. (2009) Heterotopic triplet pregnancy with bilateral tubal and intrauterine pregnancy after spontaneous conception. Eur J Obstet Gynecol Reprod Biol 142: 161-162.

20. Pan HS, Chuang J, Chiu SF, Hsieh BC, Lin YH, et al. (2002) Heterotopic triplet pregnancy: report of a case with bilateral tubal pregnancy and an intrauterine pregnancy. Hum Reprod 17: 1363-1366.

21. Hoopmann M, Wilhelm L, Possover M, Nawroth F (2003) Heterotopic triplet pregnancy with bilateral tubal and intrauterine pregnancy after IVF. Reprod Biomed Online 6: 345-348.

22. Hanf V, Dietl J, Gagsteiger F, Pfeiffer KH (1990) Bilateral tubal pregnancy with intra-uterine gestation after IVF-ET: therapy by bilateral laparoscopic salpingectomy; a case report. Eur J Obstet Gynecol Reprod Biol 37: 87-90.

23. Thakur R, El-Menabawey M (1996) Combined intra-uterine and extra-uterine pregnancy associated with mild hyperstimulation syndrome after clomiphene ovulation induction. Hum Reprod 11: 1583-1584.

24. Vanderheyden JS, van Dam PA (1987) The rising incidence of heterotopic pregnancy: two case reports. Eur J Obstet Gynecol Reprod Biol 24: 341-346.

25. Moosburger D, Tews G (1996) Severe ovarian hyperstimulation syndrome and combined intrauterine and tubal pregnancy after in-vitro fertilization and embryo transfer. Hum Reprod 11:68-69.

26. Snyder T, delCastillo J, Graff J, Hoxsey R, Hefti M (1988) Heterotopic pregnancy after in vitro fertilization and ovulatory drugs. Ann Emerg Med 17: 846-849.

27. El-Nafaty AU, Melah GS, Massa AA, Yahaya UR, Bukar M, et al. (2005) Heterotopic pregnancy in a natural conception cycle. A case report. Niger $\mathrm{J}$ Clin Pract 8: 128-129.

28. Janata J (1978) Combined intra-uterine and ectopic pregnancy following the treatment with clomiphen (author's transl). Geburtshilfe Frauenheilkd 38: 476 477.

29. Ombelet W, Van der Merwe JV (1986) Combined extra- and intra-uterine pregnancies. A report of 3 cases. S Afr Med J 70: 559-560.

30. Nugent PJ (1992) Ruptured ectopic pregnancy in a patient with a recent intrauterine abortion. Ann Emerg Med 21: 97-99.

31. Carli M, Zanini P (1989) [Coexistence of intra- and extra-uterine pregnancy: a clinical case]. Medicina (Firenze ) 9: 396-397.

32. Cheng PJ, Chueh HY, Qu JT (2004) Heterotopic pregnancy in a natura conception cycle presenting as hematometra. Obstet Gynecol 104: 1195-1198.

33. Prapas Y, Prapas N, Chatziparasidou A, Konstantinou P, Vlassis G (1994) Ovarian hyperstimulation syndrome and heterotopic pregnancy after IVF. Acta Eur Fertil 25: 331-333.

34. Quiel V (1998) [Combined intra- and extra-uterine pregnancy as a complication of ovarian stimulation and homologous insemination]. Zentralbl Gynakol 120 465-467.

35. Wittmann M (1988) [Simultaneous intra- and extrauterine pregnancy] Geburtshilfe Frauenheilkd 48: 659-660.

36. Yovich JL, McColm SC, Turner SR, Matson PL (1985) Heterotopic pregnancy from in vitro fertilization. J In Vitro Fert Embryo Transf 2: 143-150. 
Citation: Mynbaev OA, Khachatryan AK, Kosmas IP, Eliseeva MY, Khachatryan TA, et al. (2013) Case Report of Combined Twin Tubal and Intrauterine Pregnancies. J Clin Case Rep 3: 242. doi:10.4172/2165-7920.1000242

37. Oki T, Douchi T, Nakamura S, Maruta K, ljuin H, et al. (1998) A woman with three ectopic pregnancies after in-vitro fertilization and embryo transfer. Hum Reprod 13: 468-470.
38. Tandon R, Goel P, Saha PK, Devi L (2009) Spontaneous heterotopic pregnancy with tubal rupture: a case report and review of the literature. J Med Case Rep 3: 8153 . 